lectomy for acute pulmonary artery occlusion following Fontan procedure. Ann Thorac Surg 1988;45:335-6.

3. Okita Y, Miki S, Kusuhara K, et al. Massive systemic venous thrombosis after Fontan operation: report of a case. Thorac Cardiovasc Surg 1988;36:234-6.

4. Mahony L, Nikaidoh H, Fixler DE. Thrombolytic treat- ment with streptokinase for late intraatrial thrombosis after modified Fontan procedure. Am J Cardiol 1988;62: 343-4.

5. Dobell ARC, Trusler GA, Smallhorn JF, Williams WG. Atrial thrombi after the Fontan operation. Ann Thorac Surg 1986;42:664-7.

\title{
FATAL PULMONARY HYPERTENSION AFTER HEART TRANSPLANTATION: SEQUELAE OF POSTACTINIC PULMONARY VASCULAR CHANGES
}

\author{
Urs Niederhäuser, MD, ${ }^{a}$ Paul Vogt, MD, ${ }^{a}$ Jakob Schneider, MD, ${ }^{b}$ Edith R. Schmid, MD, Mico Tornic, MD, \\ Christian Gasser, MD, ${ }^{d}$ Erwin Oechslin, MD, ${ }^{\mathrm{d}}$ and Marko I. Turina, MD, ${ }^{\mathrm{a}}$ Zürich, Switzerland
}

A 41-year-old woman was admitted for heart transplantation because of doxorubicin-induced cardiomyopathy. In 1981, she had undergone combined treatment with chemotherapy and radiotherapy for Hodgkin's disease stage IIB and 9 years later for recurrent stage IIIB disease. The treatment included a total doxorubicin dose of 340 $\mathrm{mg}$ and a total irradiation dose to the paraaortic space of 3620 rads; She was in total remission for 4 years when her cardiomyopathy was in an end stage and she showed catecholamine-dependent biventricular heart failure. Cardiac catheterization revealed a severe dilative cardiomyopathy, probably caused by the toxic effects of doxorubicin. Pulmonary artery pressure was $55 / 32 / 38 \mathrm{~mm} \mathrm{Hg}$, and pulmonary vascular resistance was $331 \mathrm{dyn} \cdot \mathrm{sec} \cdot \mathrm{cm}^{-5}$. Cardiac index was $1.73 \mathrm{~L} / \mathrm{m}^{2}$.

In July 1994, orthotopic heart transplantation was performed. No residual tumor tissue was found. Before the aortic crossclamp was opened, $1000 \mathrm{mg}$ of methylprednisolone was administered as a standard immunosuppressive therapy. Total ischemic time was 77 minutes. A right-sided parietal pleurectomy was performed to drain chronic benign effusion. Because of expected diffuse bleeding after pleurectomy, 2 million units of aprotinin were added to the extracorporeal circulation (ECC). Weaning from cardiopulmonary bypass was accomplished without inotropic support. Seventeen minutes after decanulation and after initiation of heparin reversal with an infusion of protamine, a sudden rise of mean pulmonary artery pressure occurred, from 15 to 60 $\mathrm{mm} \mathrm{Hg}$. Mean arterial pressure dropped from 68 to $20 \mathrm{~mm}$ $\mathrm{Hg}$. Oxygenation remained adequate. The patient was immediately fully reheparinized, recannulated, and reperfused. All the following attempts to wean the patient from ECC failed because of a progressive rise of pulmonary artery pressure to suprasystemic levels, with maximal mean values of $90 \mathrm{~mm} \mathrm{Hg}$ and concomitant failure of the right side of the

From the Clinic for Cardiovascular Surgery, ${ }^{a}$ Department of Pathology, Institute of Anesthesiology, ${ }^{c}$ and Department of Medicine, ${ }^{d}$ University Hospital, Zürich, Switzerland.

Accepted for publication May 5, 1995.

J THORAC CARDIOVASC SURG 1995;110:1565-6

Copyright 1995 by Mosby-Year Book, Inc.

$0022-5223 / 95 \$ 5.00+0 \quad \mathbf{1 2 / 5 4 / 6 6 2 2 0}$ heart. Inotropic substances (epinephrine, dopamine, and norepinephrine) were ineffective and nitroglycerin, prostaglandin $E_{1}$, and inhaled nitric oxide had no effect on pulmonary vascular resistance. Acute pulmonary artery embolization could be excluded. Even the implanted right ventricular assist device could not overcome the pulmonary vascular resistance. At a flow rate of 2 to $3 \mathrm{~L} / \mathrm{min}$, pulmonary artery pressure was in excess of $90 \mathrm{~mm} \mathrm{Hg}$, with a systemic arterial pressure of 25 to $30 \mathrm{~mm} \mathrm{Hg}$. Inspiratory airway pressure rose to $40 \mathrm{~cm} \mathrm{H} \mathrm{H}_{2} \mathrm{O}$, and the patient died of massive hemorrhagic pulmonary edema with failing oxygenation. At autopsy, the transplanted heart showed no relevant pathologic changes. Significant alterations were found in the lungs, mostly in the venous and capillary circulation, with intravascular coagulation. The small pulmonary veins were narrowed by a loose, sparsely cellular intimal tissue suggestive of intimal swelling during operation (Fig. 1). The capillaries were filled with fibrin plugs. The lungs showed fibrosis in the pericardiac region, mostly as a result of former radiation, and a hemorrhagic edema was present. Before operation, plasma protein levels were in the normal range and immunoelectrophoresis showed only a slight hypoimmunoglobulinemia.

Adverse reaction to protamine. The patient had no predisposing factors toward adverse reactions to protamine (previous exposure to the drug, insulin dependence, fish allergy), ${ }^{1}$ and the clinical picture of isolated and overwhelming pulmonary hypertension was not compatible with an immunoglobulin E-mediated anaphylaxis, which decreases systemic vascular resistance and increases capillary permeability and airway resistance. After administration of $1 \mathrm{gm}$ of methylprednisone, any anaphylactic reaction should also be diminished. The direct systemic vasodilatory effect of protamine was obviated by very slow rates of drug administration $(5 \mathrm{mg} / \mathrm{min})$. Protamine may also react with circulating immunoglobulin $\mathrm{G}$ or immunoglobulin $\mathrm{M}$ antibodies, which results in pulmonary vasoconstriction by elevation of anaphylatoxins and thromboxane $\mathrm{A}_{2}$. Pulmonary hypertension was excessive, however, and could not be influenced by any vasodilatory drug as would be expected in thromboxane $\mathrm{A}_{2}$-related hypertension. $^{2}$ Protamine-induced pulmonary hypertension involves the arterial vascular bed mainly by increasing the tone of arterioles, whereas in our patient the pathologic substrate was a predominant obstruction of the venous circulation, which best explains the impressive hemor- 


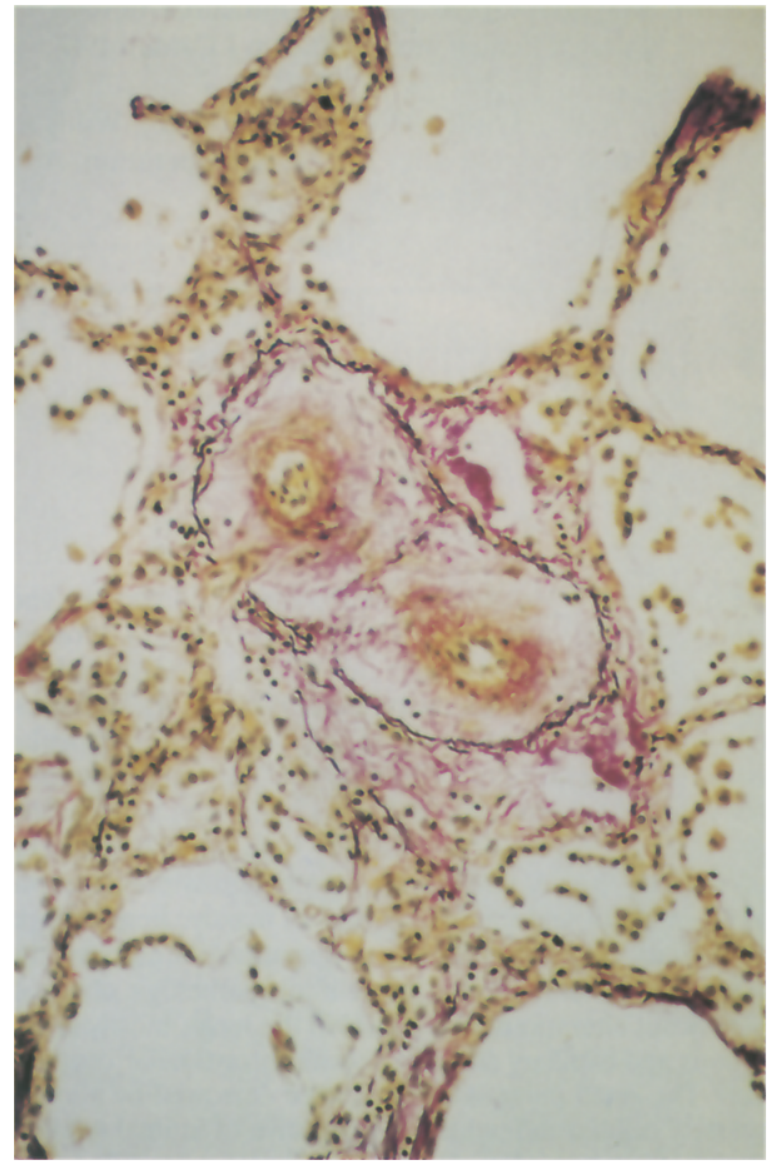

Fig. 1. Small pulmonary venules are extremely narrowed by a sparsely cellular tissue. The loose appearance is suggestive of intimal swelling during the operation (van Gieson-elastin stain).

rhagic pulmonary edema. However, overlapping symptoms of venous and arterial pulmonary hypertension were not distinguishable during the operation.

Adverse reaction to aprotinin. Aprotinin has characteristics similar to those of protamine. ${ }^{3}$ The patient was not at risk for an anaphylactic reaction because she had never had contact with that drug before. Immunoglobulin G, immunoglobulin $\mathrm{M}$, and immunoglobulin $\mathrm{E}$ antibodies could be ruled out. Moreover, the time course of the adverse reaction was not explained by complement activation, and the patient was not reactive to pulmonary vasodilators, which again would be expected in complement-dependent, thromboxane $\mathrm{A}_{2}$-induced pulmonary hypertension. Aprotinin is postulated to inhibit fibrinolysis and preserve platelet function, and it is used in cardiac operations to reduce blood loss and blood transfusion requirements. In our patient, aprotinin was indicated because of expected enhanced bleeding after additional parietal pleurectomy. Side effects have been reported with high-dose aprotinin therapy. ${ }^{4}$ Especially in older patients, aprotinin may be responsible for disseminated intravascular coagulation if used in procedures with hypothermic circulatory arrest. Only low-dose aprotinin ( 2 million units) was used in our patient. Hypothermia was moderate ( $30^{\circ} \mathrm{C}$ core temperature), and circulatory arrest was never established. Low flow in the pulmonary circulation only occurred during ECC under full heparinization. The activated clotting time was kept well above 400 seconds during the whole ECC to avoid inadequate anticoagulation.

Postactinic pulmonary vascular changes. The most striking pathologic finding in our patient was an intimal thickening and consequent luminal narrowing of small pulmonary veins, as described by Wilkinson and Maclennan ${ }^{5}$ in irradiated lungs. Radiation produces free radicals, mostly oxygen derived, and free radicals are predominantly found in the pulmonary veins, which have a higher oxygen saturation than the arteries. We believe that these postactinic and obstructing vascular changes were primarily responsible for the hypertension in the pulmonary circulation after the cessation of ECC. Reversal of heparinization by protamine and also aprotinin may have additionally contributed to hypercoagulability and to final intravascular coagulation in small pulmonary veins. Other than 2 units of fresh-frozen plasma, no other relevant coagulatory products were used. Pulmonary hypertension was fixed and did not respond to standard pulmonary vasodilators. The observed hemorrhagic pulmonary edema can be explained only by a venous form of pulmonary hypertension. The pulmonary side effects of doxorubicin and bleomycin are well known but are not explanatory in our case. The impressive swelling of the small pulmonary veins, with a luminal narrowing of up to $90 \%$, must have occurred later during the operation, because if such changes had been present before operation, they would have resulted in much higher pulmonary artery pressure. We can only speculate about the cause of this swelling, and we assume a cofactorial pathogenesis of postactinic vascular changes with bleomycin or doxorubicin toxic effects and ECC.

Conclusion. We suggest that patients with postactinic changes of the pulmonary vascular system may be at increased risk for hypertension and intravascular coagulation in the pulmonary veins. We therefore recommend extensive evaluation and careful perioperative monitoring of the coagulation state of these patients. Deep hypothermia or circulatory arrest should be avoided, as should the administration of aprotinin or even protamine for reversal of heparin. Use of a heparin-coated ECC with low-dose heparinization might be advisable. The role of preoperative lung biopsy remains uncertain.

\section{REFERENCES}

1. Horrow JC. Protamine allergy. J Cardiothorac Anesth 1988;2:225-42.

2. Lowenstein E, Johnston WE, Lappas DG, et al. Catastrophic pulmonary vasoconstriction associated withprotamin reversal of heparin. Anesthesiology 1983;59:470-3.

3. Levy JH. Antibody formation after drug administration during cardiac surgery: parameters for aprotinin use. $\mathbf{J}$ Heart Lung Transplant 1993;12:26-32.

4. Cosgrove DM, Heric B, Lytle BW, et al. Aprotinin therapy for reoperative myocardial revascularization: a placebo controlled study. Ann Thorac Surg 1992;54:1031-8.

5. Wilkinson MJ, Maclennan KA. Vascular changes in irradiated lungs: a morphometric study. J Pathol 1989; 158:229-32. 